\title{
Fundamental properties and atmospheric structure of the red supergiant VY Canis Majoris based on VLTI/AMBER spectro-interferometry ${ }^{\star, \star \star}$
}

\author{
M. Wittkowski ${ }^{1}$, P. H. Hauschildt ${ }^{2}$, B. Arroyo-Torres ${ }^{3}$, and J. M. Marcaide ${ }^{3}$ \\ 1 ESO, Karl-Schwarzschild-Str. 2, 85748 Garching bei München, Germany \\ e-mail: mwittkow@eso.org \\ 2 Hamburger Sternwarte, Gojenbergsweg 112, 21029 Hamburg, Germany \\ 3 Dpt. Astronomia i Astrofísica, Universitat de València, C/ Dr. Moliner 50, 46100 Burjassot, Spain \\ Received 28 February 2012 / Accepted 21 March 2012
}

\section{ABSTRACT}

\begin{abstract}
Aims. We investigate the atmospheric structure and fundamental properties of the red supergiant VY CMa.
Methods. We obtained near-infrared spectro-interferometric observations of VY CMa with spectral resolutions of 35 and 1500 using the AMBER instrument at the VLTI.

Results. The visibility data indicate the presence of molecular layers of water vapor and $\mathrm{CO}$ in the extended atmosphere with an asymmetric morphology. The uniform disk diameter in the water band around $2.0 \mu \mathrm{m}$ is increased by $\sim 20 \%$ compared to the nearcontinuum bandpass at $2.20-2.25 \mu \mathrm{m}$, and in the $\mathrm{CO}$ band at $2.3-2.5 \mu \mathrm{m}$ it is increased by up to $\sim 50 \%$. The closure phases indicate relatively small deviations from point symmetry close to the photospheric layer, and stronger deviations in the extended $\mathrm{H}_{2} \mathrm{O}$ and $\mathrm{CO}$ layers. Making use of the high spatial and spectral resolution, a near-continuum bandpass can be isolated from contamination by molecular and dusty layers, and the Rosseland-mean photospheric angular diameter is estimated to $11.3 \pm 0.3$ mas based on a PHOENIX atmosphere model. Together with recent high-precision estimates of the distance and spectro-photometry, this estimate corresponds to a radius of $1420 \pm 120 R_{\odot}$ and an effective temperature of $3490 \pm 90 \mathrm{~K}$.

Conclusions. VY CMa exhibits asymmetric, possibly clumpy, atmospheric layers of $\mathrm{H}_{2} \mathrm{O}$ and $\mathrm{CO}$, which are not co-spatial, within a larger elongated dusty envelope. Our revised fundamental parameters put VY CMa close to the Hayashi limit of recent evolutionary tracks of initial mass $25 M_{\odot}$ with rotation or $32 M_{\odot}$ without rotation, shortly before evolving blueward in the HR-diagram.
\end{abstract}

Key words. supergiants - stars: atmospheres - stars: fundamental parameters - stars: mass-loss - stars: individual: VY CMa

\section{Introduction}

VY Canis Majoris (VY CMa) is one of the most luminous and most massive red supergiants, and thus most likely progenitors of a core-collapse supernova (SN) in our Galaxy. Because of its brightness and importance, it has traditionally been extensively observed at many wavelengths. Currently, VY CMa experiences a renewed and increased interest because of observations at new facilities including, for instance, the Herschel Space Observatory (Royer et al. 2010) and the submillimeter array (Fu et al. 2012), as well as because of new results regarding the complexity of its molecular envelope (e.g., Ziurys et al. 2007).

While is has been clear that VY CMa's fundamental parameters are extreme, their exact values have been debated during the last decade with luminosities between $6 \times 10^{4} L_{\odot}$ and $5 \times 10^{5} L_{\odot}$, effective temperatures between $2700 \mathrm{~K}$ and $3650 \mathrm{~K}$, radii between $600 R_{\odot}$ and $3000 R_{\odot}$, and initial masses between $12 M_{\odot}$ and $40 M_{\odot}$ (Smith et al. 2001; Monnier et al. 2000, 2004; Massey et al. 2006). Very recently, precise parallax measurements by Choi et al. (2008) and Zhang et al. (2012) significantly improved the distance estimate to about $1.2 \mathrm{kpc}$, which together with recent accurate broad-band photometry by Smith et al. (2001) points to a luminosity of about $3 \times 10^{5} L_{\odot}$.

\footnotetext{
* Based on observations made with the VLT Interferometer (VLTI) at Paranal Observatory under programme ID 386.D-0012.

$\star \star$ Figures 2, 3 and 5 are available in electronic form at http://www . aanda.org
}

VY CMa is embedded in a large optical nebula (Herbig 1972) and is also an extended object in the infrared (McCarthy \& Low 1975). Several studies have investigated the asymmetric circumstellar environment in detail and revealed a complex asymmetric morphology (e.g. Wittkowski et al. 1998; Monnier et al. 1999, 2000, 2004; Smith et al. 2001; Muller et al. 2007; Humphreys et al. 2007; Jones et al. 2007). Recently, Smith et al. (2009) proposed a scenario of VY CMa's circumstellar environment that consists of fast and dense $\mathrm{CO} /$ dust cloudlets that are ejected into a larger relatively slow and less dense asymmetric envelope due to a strong and variable mass loss.

\section{Observations and data reduction}

We observed VY CMa with the VLTI/AMBER instrument in visitor mode on 6 and 7 March 2011. Table 1 lists the details of our observations. We used the E0-G0-H0 configuration of the VLTI, providing projected baseline lengths between $11.2 \mathrm{~m}$ and $48.0 \mathrm{~m}$. We obtained 5 observations using the low resolution mode with a spectral resolution of $R \sim 30$ at different hour angles, thus different projected baseline lengths and angles. We also obtained one observation using each of the two $K$-band medium resolution modes, together covering wavelengths between $1.92 \mu \mathrm{m}$ and $2.47 \mu \mathrm{m}$ at a spectral resolution of $R \sim 1500$. All VY CMa observations are embedded between observations of an interferometric calibration star. We chose HR 3052 (spectral type K5 III, angular diameter $2.62 \pm 0.19$ mas) and 1 Pup (K5 III, $3.85 \pm 0.28$ mas) from the 
Table 1. Log of our VLTI/AMBER observations.

\begin{tabular}{lccccc}
\hline \hline Target & $\begin{array}{c}\text { Date } \\
2011-03\end{array}$ & $\begin{array}{c}\text { Time } \\
\text { UT }\end{array}$ & Mode & $\begin{array}{c}\text { Baseline } \\
\mathrm{m}\end{array}$ & $\begin{array}{c}\text { PA } \\
\text { deg }\end{array}$ \\
\hline VY CMa & -06 & $1: 42$ & Low-JHK & $15.8 / 31.6 / 47.5$ & -75 \\
VY CMa & -06 & $3: 05$ & MR-K 2.3 & $14.4 / 28.7 / 43.0$ & -83 \\
VY CMa & -06 & $4: 14$ & Low-JHK & $11.8 / 23.6 / 35.4$ & -90 \\
VY CMa & -07 & $0: 55$ & Low-JHK & $16.0 / 32.0 / 48.0$ & -70 \\
VY CMa & -07 & $1: 35$ & Low-JHK & $15.9 / 31.7 / 47.6$ & -75 \\
VY CMa & -07 & $3: 02$ & Low-JHK & $14.3 / 28.6 / 42.9$ & -83 \\
VY CMa & -07 & $4: 29$ & MR-K 2.1 & $11.2 / 22.3 / 33.5$ & -89 \\
\hline
\end{tabular}

Notes. The AMBER instrument modes are Low-JHK (range 1.2$2.5 \mu \mathrm{m}$, spectral resolution $R \sim 30)$, MR-K $2.1(1.92-2.26 \mu \mathrm{m}, R \sim$ 1500), and MR-K 2.3 (2.12-2.47 $\mu \mathrm{m}, R \sim 1500)$. The baseline denotes the projected baseline lengths, and the position angle (PA) the projected position angle on sky (east of north).

ESO Calibrator Selector CalVin based on the catalog by Lafrasse et al. (2010).

We obtained raw visibility and closure phase using the amdlib data reduction package (Tatulli et al. 2007; Chelli et al. 2009), version 3.0.3. We performed an absolute wavelength calibration as in Wittkowski et al. (2011). Calibrated visibility spectra were obtained by using an average of two transfer function measurements taken before and after each science observation.

Finally, we took into account that VY CMa shows an extended dust shell that exceeds the AMBER field of view (FoV). In this case, the calibrated visibility values are normalized to the flux within the AMBER FoV and not to the larger flux of the more extended target, which increases the fraction of the correlated flux and thus the visibility values (cf. Driebe et al. 2009). Here, this scaling factor was estimated using the results by Wittkowski et al. (2008) based on bispectrum speckle interferometry with a sufficiently large FoV. They obtained a relative flux contribution of an unresolved component of $9 \% / 50 \%$ at wavelengths of $1.28 \mu \mathrm{m} / 2.17 \mu \mathrm{m}$. With our longer baselines, their extended component is fully resolved, and our visibility data describe their unresolved component. Thus, we used a model of a central uniform disk (UD) and a fully resolved component, and scaled our visibility values such that we obtained the same relative flux contribution of our central component. The resulting scale factor for the visibility modulus was $0.45 / 0.90$ in the $J / K$ bands, and we used an interpolated $H$-band value of 0.65 . We note that this procedure is applied in order to remove the instrumental signature of the limited FoV, which may be useful for a future combination of our data with data obtained by other instruments. However, the choice of the scaling factor does not affect the results presented in the following sections, except for the fraction of the flux that is attributed to the central stellar component, as the visibilities are fitted with a two-component model where the flux ration is a free parameter (see Sect. 4).

\section{Atmospheric structure and morphology}

Figure 1 shows the resulting $H$ - and $K$-band squared visibility amplitudes and closure phases of VY CMa obtained with the low resolution mode of the AMBER instrument. The $J$ band values are not shown because of their lower quality. Also indicated are the positions of $\mathrm{H}_{2} \mathrm{O}$ and $\mathrm{CO}$ bands after Lançon \& Wood (2000). The visibility curves show different heights, corresponding to the different projected baseline lengths. All curves exhibit a characteristic bumpy shape that resembles that of recent AMBER observations of Mira variables and that are interpreted as being indicative of the presence of molecular layers lying above the continuum-forming
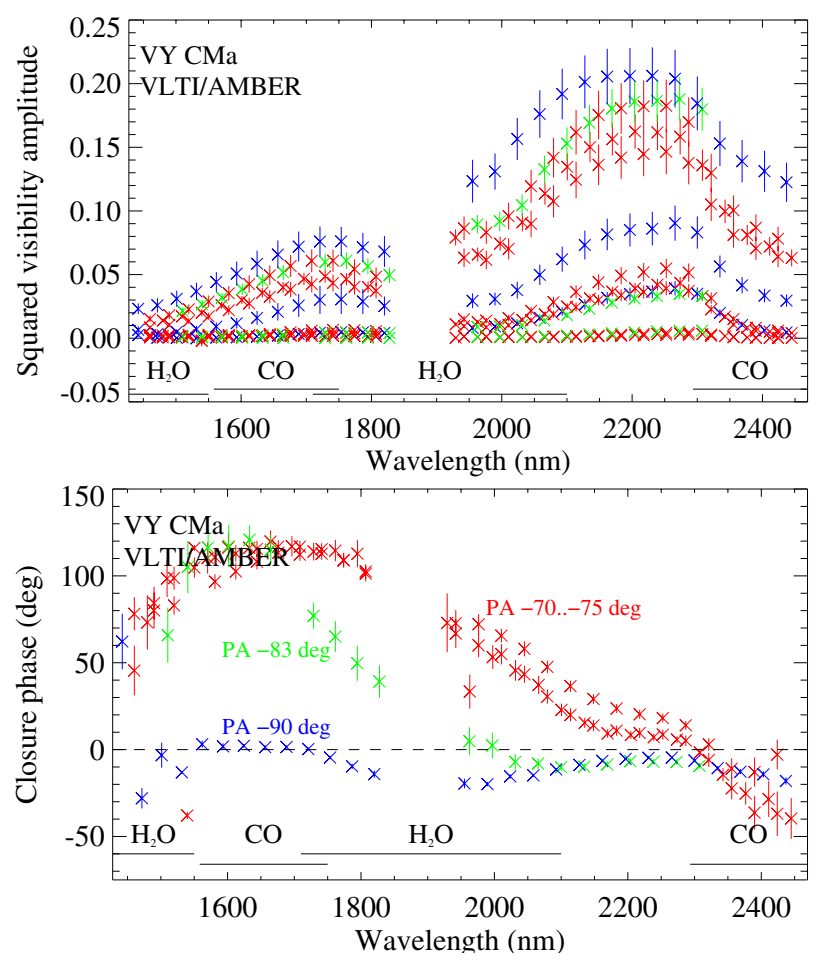

Fig. 1. Squared visibility amplitudes (top) and closure phases (bottom) of VY CMa obtained with the low resolution mode of the AMBER instrument. Also shown are the positions of $\mathrm{CO}$ and $\mathrm{H}_{2} \mathrm{O}$ bands after Lançon \& Wood (2000). The different colors indicate our different ranges of PA (cf. Table 1).

photosphere (Wittkowski et al. 2008, 2011). Our VY CMa visibility curves show maxima near $2.25 \mu \mathrm{m}$ and $1.70 \mu \mathrm{m}$, where the intensity of molecular bands is lowest. The visibility decreases toward the water bands centered at $\sim 1.5 \mu \mathrm{m}$ and $\sim 1.9 \mu \mathrm{m}$. It also exhibits a steep drop at the onset of the $\mathrm{CO}$ absorption feature at $2.3 \mu \mathrm{m}$. The decrease of the visibility indicates an increased contribution from extended intensity at these molecular bands. The presence of molecular layers in the extended atmospheres of red supergiants is consistent with earlier observations of red supergiants such as $\mu$ Cep and Betelgeuse (Perrin et al. 2004, 2005; Tsuji 2006) and with the detection of warm water layers in the ISO and Herschel spectra of VY CMa (Polehampton et al. 2010; Royer et al. 2010).

Our VY CMa closure phases exhibit complex curves that correlate with the positions of the molecular bands and with the projected position angles of the observation. Non-0/non180 deg closure phase values are indicative of deviations from point symmetry. At the near-continuum band close to $2.25 \mu \mathrm{m}$ all curves show relatively small closure phases with absolute values $<20$ deg. This indicates relatively small deviations from point symmetry of atmospheric layers close to the photosphere. These deviations may be caused by photospheric convection cells or by asymmetric molecular emission that may not be completely absent at a near-continuum bandpass. At the molecular bands of $\mathrm{H}_{2} \mathrm{O}$ and $\mathrm{CO}$, the closure phase values increase, which indicates a stronger deviation from point symmetry, and also show variations as a function of position angle. Along the EW orientation (PA $-90 \mathrm{deg}$ ), the closure phase values remain at absolute values below $\sim 20 \mathrm{deg}$ within the $\mathrm{CO}$ band and the water band. Toward the NW-SE orientation (position angle $-70 \mathrm{deg}$ ), they show a $\sim 90$ deg signal at the center of the water band at $1.9 \mu \mathrm{m}$, and absolute values up to $\sim 40 \mathrm{deg}$ at the CO band beyond $2.3 \mu \mathrm{m}$. The increased asymmetry of the molecular layers 
from the E-W direction toward the NW-SE direction coincides with the larger-scale asymmetry of the dusty circumstellar envelope (PA $160 \mathrm{deg}$ ) reported by Wittkowski et al. (1998) at a wavelength of $2.17 \mu \mathrm{m}$. Along the PA of the stronger asymmetry, the sign of the closure phase differs between the $\mathrm{H}_{2} \mathrm{O}$ band $\mathrm{CO}$ band indicating that the $\mathrm{H}_{2} \mathrm{O}$ and $\mathrm{CO}$ regions are not co-spatial.

Figures 2 and 3 (electronic edition) show the results obtained with the medium resolution modes at PA $-89 \mathrm{deg}$ and $-83 \mathrm{deg}$. These results are consistent with the low resolution results shown in Fig. 1. The visibility shows a maximum at the near-continuum bandpass at $\sim 2.20-2.25 \mu \mathrm{m}$ and decreases toward the water band centered at $1.9 \mu \mathrm{m}$ and the $\mathrm{CO}$ lines between $2.3 \mu \mathrm{m}$ and $2.5 \mu \mathrm{m}$, in particular at the $\mathrm{CO}$ band-heads. The corresponding UD curve shows minimum extensions at 2.20$2.25 \mu \mathrm{m}$ and increases by about $20 \%$ at the water band near $2.0 \mu \mathrm{m}$ and by up to $\sim 50 \%$ at the $\mathrm{CO}$ band-heads. The closure phase shows relatively small absolute values of about $10 \mathrm{deg}$ at the near-continuum bandpass, of 30-40 deg at the water and CO bands, and peaks at the $\mathrm{CO}$ band-heads, confirming asymmetric structures of extended CO band-head emission. Also shown in Figs. 2 and 3 are visibility fits of a UD model and a PHOENIX model atmosphere to the $2.20-2.25 \mu \mathrm{m}$ near-continuum band, as described in the following section.

Overall, our data may qualitatively be consistent with the scenario proposed by Smith et al. (2009), consisting of a conelike envelope toward the SE direction that extends from a largescale dusty envelope to a small-scale molecular water and $\mathrm{CO}$ envelope close to the photosphere, and additional close $\mathrm{CO}$ cloudlets that may not be co-spatial with the water envelope. However, for a definite and quantitative picture of the inner molecular envelope of VY CMa, an interferometric imaging campaign using a well-filled $u v$-plane will be required.

\section{Fundamental parameters}

The most direct method to derive the effective temperature is a measurement of the radius where the Rosseland-mean optical depth equals $2 / 3$ together with a measurement of the bolometric flux. The luminosity is most directly estimated by measurements of the bolometric flux and the distance. The bolometric flux of VY CMa can be well estimated using the highprecision photometry by Smith et al. (2001) complemented by the IRAS fluxes (Beichman et al. 1988), cf. also Jones et al. (2007) and Choi et al. (2008). We de-reddened these data using an $E_{B-V}$ value of 0.6 obtained from the COBE maps (Schlegel et al. 1998), and obtained an integrated bolometric flux of $f_{\text {bol }}=(6.3+/-0.3) 10^{-13} \mathrm{~W} / \mathrm{cm}^{2}$. The distance estimate to VY CMa has recently been improved by high precision parallax measurements by Choi et al. (2008) and Zhang et al. (2012). Here we use the average of these two measurements, and adopt a distance of $d=1.17_{-0.07}^{+0.08} \mathrm{kpc}$. The classic distance used most often in the literature was $1.5 \mathrm{kpc}$ going back to Lada \& Reid (1978) and the suspected membership to the open cluster NGC 2362 , but whose distance has also been revised from $1.5 \mathrm{kpc}$ to $1.2 \mathrm{kpc}$ (Mel'nik \& Dambis 2009). Our adopted bolometric flux and distance correspond to a luminosity of $L=(2.7 \pm 0.4) 10^{5} L_{\odot}$. This value is slightly smaller than recent estimates by Choi et al. (2008) and Mauron \& Josselin (2011), mostly because of the slightly larger adopted distance taking into account the additional measurement by Zhang et al. (2012), but otherwise well consistent. Choi et al. (2008) discussed that this value is also consistent with most earlier values available in the literature, if they are re-scaled to the same distance. We note that the error of $L$ is still dominated by the uncertainty of the distance.

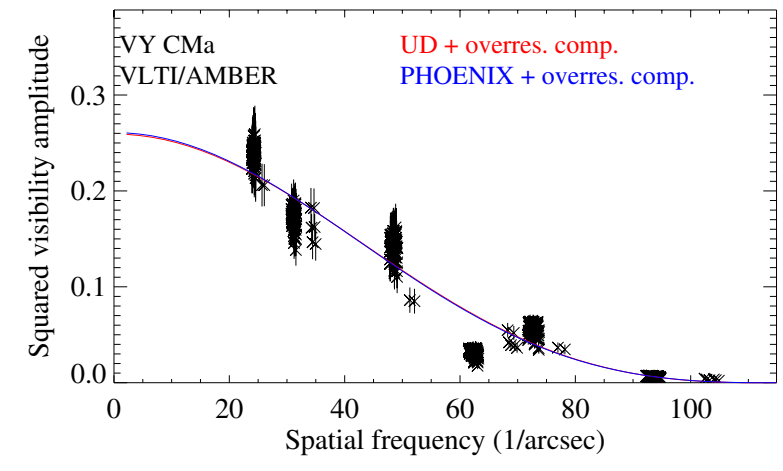

Fig. 4. Squared visibility amplitudes of VY CMa as a function of spatial frequency obtained at the near-continuum bandpass at $2.20-2.25 \mu \mathrm{m}$. Also shown are fits of a UD (red) and a PHOENIX atmosphere model (blue). These contribute $50 \%$ to the total flux, and the remaining part is attributed to an over-resolved dust component.

The angular diameter of VY CMa was measured by Monnier et al. (2004) in the $K$-band $(2.2 \pm 0.3 \mu \mathrm{m})$ to $(18.7 \pm 0.3 \pm 0.4)$ mas. They note that the relation of this diameter to a true photospheric diameter relies on additional assumption on the limb-darkening, and they also mention in Massey et al. (2006) that this value may overestimate the photospheric diameter because of a possible contamination by more extended molecular layers and dusty features. Our spectrally resolved visibility data in Figs. 1-3 confirm the presence of extended layers of $\mathrm{H}_{2} \mathrm{O}$ and $\mathrm{CO}$, so that a broad-band diameter estimate is indeed significantly contaminated by extended molecular layers and thus overestimates the photospheric diameter. With our high spectral resolution, however, we are able to estimate a nearcontinuum diameter at $2.20-2.25 \mu \mathrm{m}$ that is largely free from contamination by molecular layers. With our longer baselines (up to $48 \mathrm{~m}$ compared to $38 \mathrm{~m}$ ), our data also resolve out possible compact dusty features to smaller scales and probe spatial frequencies close to the visibility null, which significantly reduces calibration uncertainties. Figure 4 shows all our visibility data taken at spectral channels in the near-continuum bandpass between $2.20 \mu \mathrm{m}$ and $2.25 \mu \mathrm{m}$ as a function of spatial frequency, together with fits of a UD and a PHOENIX model atmosphere. The PHOENIX model was computed with version 16.03 .01 of the PHOENIX code (Hauschildt \& Baron 1999). We used solar metallicity, mass $M=20 M_{\odot}, T_{\text {eff }}=3500 \mathrm{~K}, \log g=-0.6^{1}$. We used different outer pressure boundary conditions of $10^{-7} \mathrm{dyn} / \mathrm{cm}^{2}$ and $10^{-12} \mathrm{dyn} / \mathrm{cm}^{2}$, giving virtually identical results. The bestfit diameter using the UD model was $\Theta_{U D}=(11.0 \pm 0.3)$ mas, and using the PHOENIX model $\Theta_{\text {Ross }}=(11.3 \pm 0.3)$ mas (corresponding to the model layer where $\tau_{\text {Ross }}=2 / 3$ ). Variations of the model mass, $T_{\text {eff }}$, and $\log g$ lead to diameter variations of less than 0.1 mas. The flux contribution of the stellar source was a free parameter resulting in a value of $51 \%$. This parameter accounts for the fraction of an over-resolved flux component within our FoV and also for any residual of the uncorrelated flux outside the FoV (Sect. 2). The scatter of the visibility values in Fig. 4 may be caused by calibration uncertainties (in particular the points at $60-65 / \operatorname{arcsec}$ ), or by different contributions of an over-resolved dusty component at different position angles. However, the diameter remains well constrained by the measurements close to the visibility null. Figure 5 (electronic edition) shows the corresponding fit using a near-continuum bandpass in the $H$-band at $1.70-1.75 \mu \mathrm{m}$, resulting in a well consistent

1 We computed a large model grid for different values of $M, T_{\text {eff }}$, and $\log g$, which will be presented in detail in a forthcoming publication. 


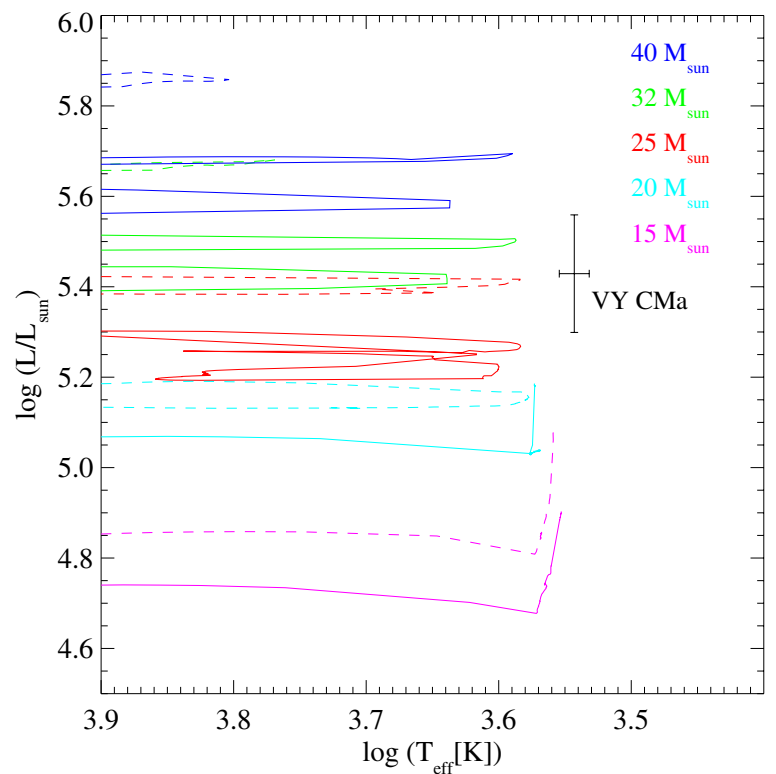

Fig. 6. Location of VY CMa's revised fundamental parameters in the HR diagram. Also shown are evolutionary tracks from Ekström et al. (2012) for masses of $15 M_{\odot}, 20 M_{\odot}, 25 M_{\odot}, 32 M_{\odot}$, and $40 M_{\odot}$. The solid lines denote the models without rotation, and the dashed lines those with rotation.

angular diameter of $\Theta_{\text {Ross }}=(11.4 \pm 0.3)$ mas, which increases the confidence in our diameter estimate. Figures 2 and 3 also indicate the UD and PHOENIX models compared to the spectrally resolved visibility data. The PHOENIX model curve is very close to a UD model, and does not predict the large extensions of the molecular layers. PHOENIX models predict near-infrared spectra of red supergiants reasonably well (Lançon et al. 2007, and Figs. 2 and 3), which likely means that opacities from molecular layers are reasonably well included, but that the extension of the model atmosphere is currently too compact. This effect is most likely caused by missing pulsation and/or wind acceleration. Since our estimate of $\Theta_{\text {Ross }}$ is based on a near-continuum bandpass, where the contamination by molecular layers is small, we do not expect this to strongly affect our estimate of the photospheric diameter. However, we can not exclude that extended molecular layers also contribute at our near-continuum bandpass to a small extent, and that our estimate of $\Theta_{\text {Ross }}$ may still overestimate the photospheric diameter to a small degree (of the order of $1 \sigma$ ), so that it may represent an upper limit of the true photospheric diameter. The resulting effective temperature, based on $\Theta_{\text {Ross }}$ and $f_{\text {bol }}$, is $T_{\text {eff }}=3490 \pm 90 \mathrm{~K}$. Using the recent calibration by van Belle et al. (2009), this value corresponds to a spectral type of M4, which is consistent with the spectral classification as M3/M4 by Houk \& Smith-Moore (1988) as used in the Simbad database. If $\Theta_{\text {Ross }}$ is considered an upper limit, the estimate of $T_{\text {eff }}$ is a lower limit.

Figure 6 compares the resulting position of VY CMa in the Hertzsprung-Russell (HR) diagram to recent evolutionary sequences by Ekström et al. (2012). It is located close to the red limit of recent evolutionary tracks of initial mass $25 M_{\odot}$ with rotation (current mass $15 M_{\odot}$ ) or $32 M_{\odot}$ without rotation (current mass $19 M_{\odot}$ ). The position of VY CMa is drawn slightly to the right of the Hayashi limit. This may be caused by VY CMa not being in full hydrostatic equilibrium. However, a slight remaining underestimate of $T_{\text {eff }}$, which we can not fully exclude,
Table 2. Revised fundamental properties of VY CMa.

\begin{tabular}{lcc}
\hline \hline Parameter & Value & Reference \\
\hline Distance $(\mathrm{kpc})$ & $1.17 \pm 0.08$ & 1 \\
Bolometric flux $\left(10^{-13} \mathrm{~W} / \mathrm{cm}^{2}\right)$ & $6.3 \pm 0.3$ & 2 \\
Rosseland angular diameter $(\mathrm{mas})$ & $11.3 \pm 0.3$ & 3 \\
Rosseland radius $\left(R_{\odot}\right)$ & $1420 \pm 120$ & 4 \\
Effective temperature $(\mathrm{K})$ & $3490 \pm 90$ & 5 \\
Spectral type & $\mathrm{M} 4(\mathrm{M} 3-\mathrm{M} 4.5)$ & 6 \\
Luminosity $\left(10^{5} L_{\odot}\right)$ & $2.7 \pm 0.4$ & 7 \\
Initial mass $\left(M_{\odot}\right)$ & $25 \pm 10$ & 8 \\
Current mass $\left(M_{\odot}\right)$ & $17 \pm 8$ & 9 \\
Surface gravity $(\log g / \mathrm{cgs})$ & $-0.6 \pm 0.4$ & 10 \\
\hline
\end{tabular}

References. 1: Average of Choi et al. (2008) and Zhang et al. (2012); 2: Photometry from Smith et al. (2001) and IRAS fluxes; 3: this work; 4: 3 with 1; 5: 3 with 2; 6: 5 with van Belle et al. (2009); 7: 2 with 1; 8-10: 5 and 7 with evolutionary tracks from Ekström et al. (2012).

could also explain this discrepancy. Table 2 summarizes our revised fundamental properties of VY CMa. With these parameters, VY CMa would most likely not explode at its current stage as a red supergiant, but be at a stage before moving blueward in the HR diagram.

Acknowledgements. This research has made use of the AMBER data reduction package of the Jean-Marie Mariotti Center.

\section{References}

Beichman, C. A., Neugebauer, G., Habing, H. J., Clegg, P. E., \& Chester, T. J. 1988, Infrared Astronomical Satellite (IRAS) catalogs and atlases Chelli, A., Utrera, O. H., \& Duvert, G. 2009, A\&A, 502, 705

Choi, Y. K., Hirota, T., Honma, M., et al. 2008, PASJ, 60, 1007

Driebe, T., Groh, J. H., Hofmann, K.-H., et al. 2009, A\&A, 507, 301

Ekström, S., Georgy, C., Eggenberger, P., et al. 2012, A\&A, 537, A146 Fu, R., Moullet, A., Patel, N. A., et al. 2012, ApJ, 746, 42

Hauschildt, P. H., \& Baron, E. 1999, J. Comput. Appl. Math., 109, 41 Herbig, G. H. 1972, ApJ, 172, 375

Houk, N., \& Smith-Moore, M. 1988, Michigan Catalogue of Two-dimensional Spectral Types for the HD Stars, 4

Humphreys, R. M., Helton, L. A., \& Jones, T. J. 2007, AJ, 133, 2716

Jones, T., Humphreys, R., Helton, L., Gui, C., \& Huang, X. 2007, AJ, 133, 2730

Lada, C. J., \& Reid, M. J. 1978, ApJ, 219, 95

Lafrasse, S., Mella, G., Bonneau, D., et al. 2010, Proc. SPIE, 7734, 140

Lançon, A., \& Wood, P. R. 2000, A\&AS, 146, 217

Lançon, A., Hauschildt, P. H., Ladjal, D., \& Mouhcine, M. 2007, A\&A, 468, 205

Massey, P., Levesque, E. M., \& Plez, B. 2006, ApJ, 646, 1203

Mauron, N., \& Josselin, E. 2011, A\&A, 526, A156

McCarthy, D. W., \& Low, F. J. 1975, ApJ, 202, L37

Mel'nik, A. M., \& Dambis, A. K. 2009, MNRAS, 400, 518

Monnier, J. D., Tuthill, P. G., Lopez, B., et al. 1999, ApJ, 512, 351

Monnier, J. D., Danchi, W. C., Hale, D. S., et al. 2000, ApJ, 543, 861

Monnier, J. D., Millan-Gabet, R., Tuthill, P. G., et al. 2004, ApJ, 605, 436

Muller, S., Dinh-V-Trung, Lim, J., et al. 2007, ApJ, 656, 1109

Perrin, G., Ridgway, S. T., Coudé du Foresto, V., et al. 2004, A\&A, 418, 675

Perrin, G., Ridgway, S. T., Verhoelst, T., et al. 2005, A\&A, 436, 317

Polehampton, E. T., Menten, K. M., van der Tak, F. F. S., \& White, G. J. 2010 A\&A, 510, A80

Royer, P., Decin, L., Wesson, R., et al. 2010, A\&A, 518, L145

Schlegel, D. J., Finkbeiner, D. P., \& Davis, M. 1998, ApJ, 500, 52

Smith, N., Humphreys, R. M., Davidson, K., et al. 2001, AJ, 121, 1111

Smith, N., Hinkle, K. H., \& Ryde, N. 2009, AJ, 137, 3558

Tatulli, E., Millour, F., Chelli, A., et al. 2007, A\&A, 464, 29

Tsuji, T. 2006, ApJ, 645, 1448

van Belle, G. T., Creech-Eakman, M. J., \& Hart, A. 2009, MNRAS, 394, 1925

Wittkowski, M., Langer, N., \& Weigelt, G. 1998, A\&A, 340, L39

Wittkowski, M., Boboltz, D. A., Driebe, T., et al. 2008, A\&A, 479, L21

Wittkowski, M., Boboltz, D. A., Ireland, M., et al. 2011, A\&A, 532, L7

Zhang, B., Reid, M. J., Menten, K. M., \& Zheng, X. W. 2012, ApJ, 744, 23

Ziurys, L., Milam, S., Apponi, A., \& Woolf, N. 2007, Nature, 447, 1094

Page 5 is available in the electronic edition of the journal at http: //www . aanda.org 
M. Wittkowski et al.: Fundamental properties of VY CMa
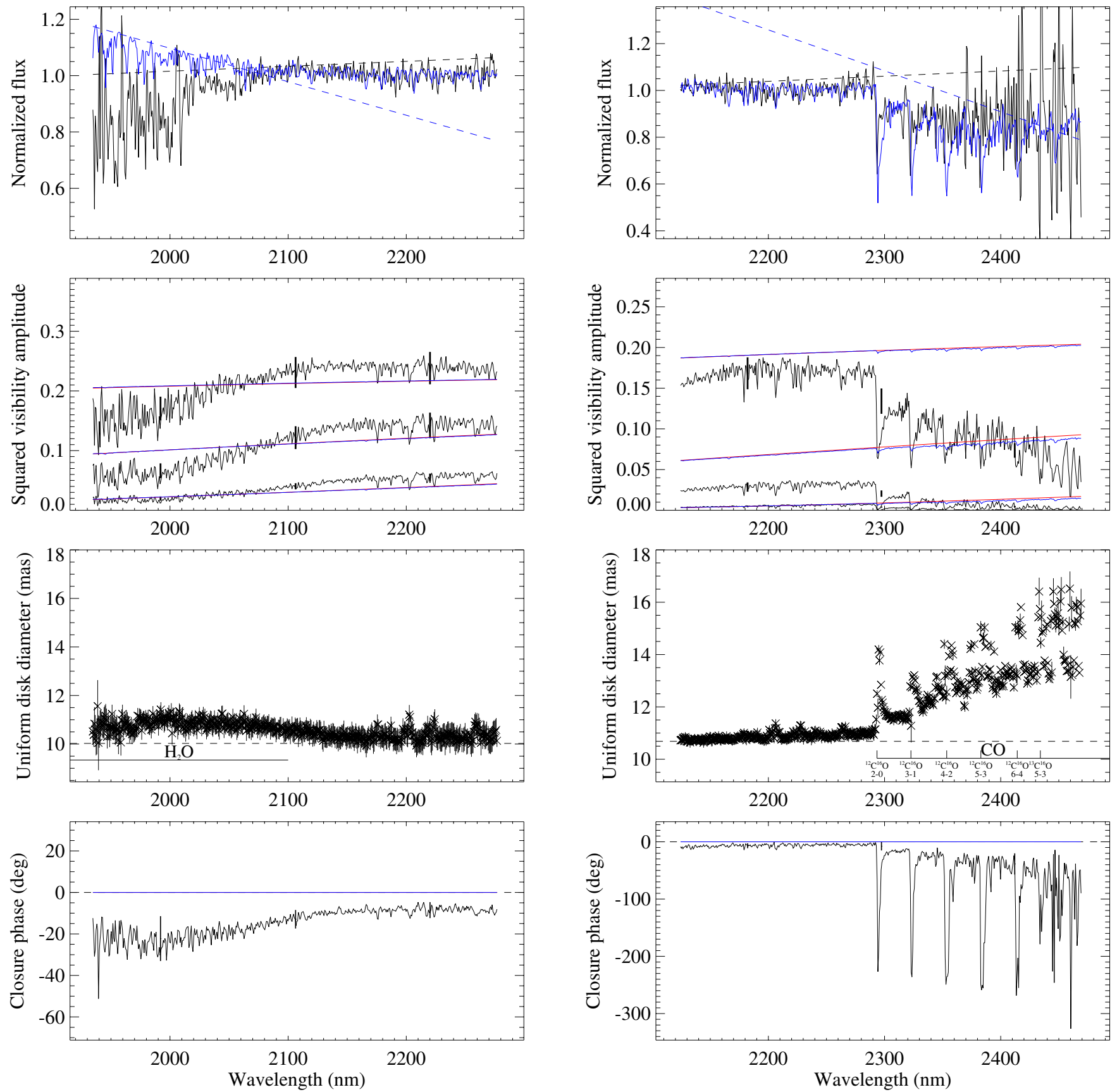

Fig. 2. Normalized flux, squared visibility amplitudes, UD diameters, and closure phases (from top to bottom) of VY CMa obtained with the MR-K $2.1 \mu \mathrm{m}$ mode (PA $-89 \mathrm{deg}$ ). The top panels also show fits of a UD (red) and a PHOENIX atmosphere model (blue). In these model visibility fits, the stellar component contributes $\sim 50 \%$ of the total flux, and the remaining part is attributed to an over-resolved dust shell. The observed and model flux spectra show different slopes because of the additional dust shell that is not present in the model, and we have subtracted a best-fit linear function for a better comparison of the spectral features. The slopes that have been compensated are indicated by the dashed lines.
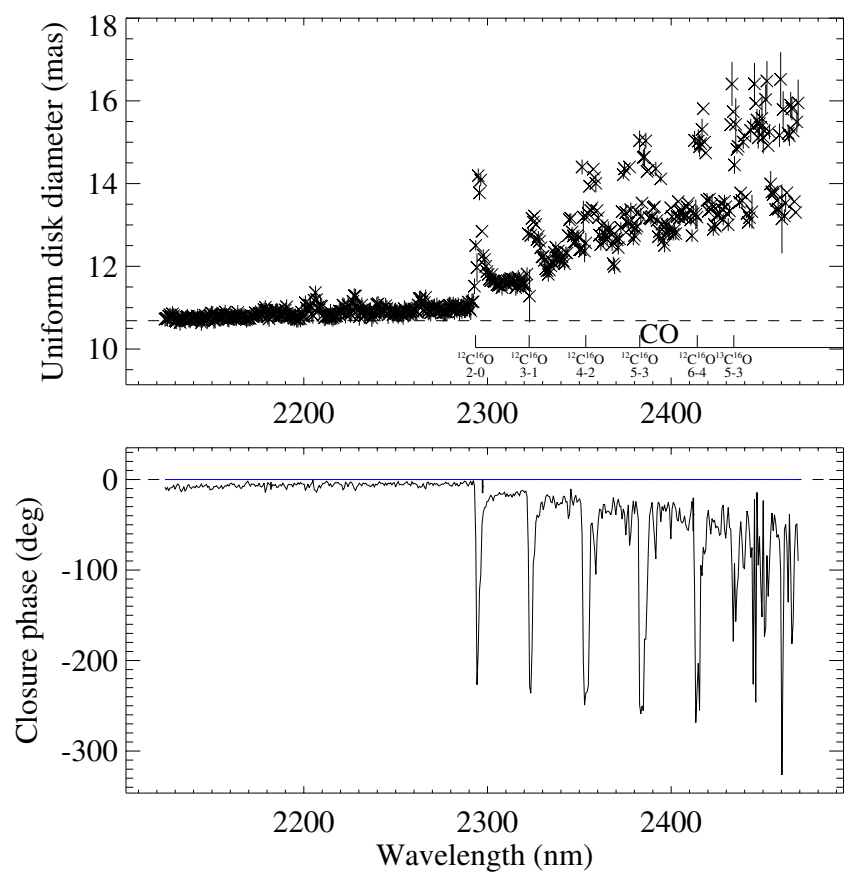

Fig. 3. As Fig. 2, but for the MR-K $2.3 \mu$ m mode (PA $-89 \mathrm{deg}$ ).

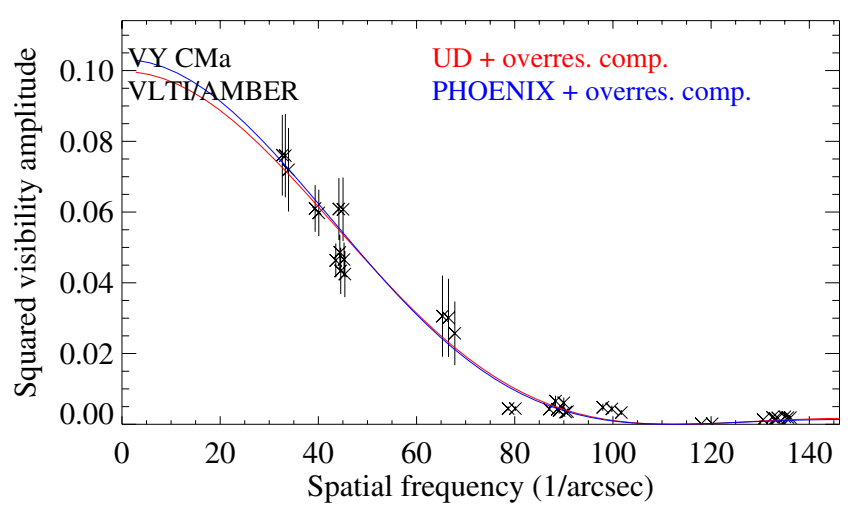

Fig. 5. As Fig. 4, but for a near-continuum bandpass in the $H$-band at $1.70-1.75 \mu \mathrm{m}$. 